\title{
Black-hole evaporation, cosmic censorship, and a quantum lower bound on the Bekenstein-Hawking temperature
}

\author{
Shahar Hod ${ }^{1,2, a}$ \\ ${ }^{1}$ The Ruppin Academic Center, 40250 Emeq Hefer, Israel \\ 2 The Hadassah Academic College, 91010 Jerusalem, Israel
}

Received: 20 June 2018 / Accepted: 31 July 2018 / Published online: 9 August 2018

(C) The Author(s) 2018

\begin{abstract}
The semi-classical Hawking evaporation process of Reissner-Nordström black holes is analyzed. It is shown that this quantum mechanism may turn a near-extremal black-hole spacetime with $T_{\mathrm{BH}} \lesssim \hbar^{2} / G^{2} M^{3}$ into an horizonless naked singularity, thus violating the Penrose cosmic censorship conjecture. It is therefore conjectured that, within the framework of a self-consistent quantum theory of gravity, the Bekenstein-Hawking temperature should be bounded from below by the simple relation $T_{\mathrm{BH}} \gtrsim \hbar^{2} / G^{2} M^{3}$.
\end{abstract}

\section{Introduction}

Following Bekenstein's proposal that black holes have a well defined entropy [1], Hawking, using a semi-classical analysis [2], has revealed the intriguing fact that these fundamental objects of general relativity are characterized by thermally distributed (filtered black-body) radiation spectra. In particular, the emission spectra of the canonical family of charged and rotating Kerr-Newman black holes are characterized by the well defined Bekenstein-Hawking temperature (We shall use natural units in which $c=k_{\mathrm{B}}=1$ )

$T_{\mathrm{BH}}=\frac{\hbar\left(r_{+}-r_{-}\right)}{4 \pi G\left(r_{+}^{2}+a^{2}\right)}$.

Here

$r_{ \pm}=G M \pm\left(G^{2} M^{2}-G Q^{2}-a^{2}\right)^{1 / 2}$,

are the (inner and outer) horizon radii which characterize the black-hole spacetime, where $\{M, Q, a\}$ are respectively the asymptotically measured black-hole mass, electric charge, and angular momentum per unit mass.

Inspection of the functional expression (1) for $T_{\mathrm{BH}}=$ $T_{\mathrm{BH}}(M, Q, a)$ immediately reveals the intriguing fact that

a e-mail: shaharhod@gmail.com the semi-classical Bekenstein-Hawking temperature approaches zero in the $M^{2}-Q^{2}-a^{2} \rightarrow 0$ limit of nearextremal black holes.

In the present paper we shall address the following physically interesting question: Can the Bekenstein-Hawking temperature of a given mass black hole be made arbitrarily small? According to Page [3,4], the answer to this question is 'yes'. Page has based his argument for the non-existence of a lower bound on the Bekenstein-Hawking temperature on the fact that, for near-extremal Reissner-Nordström $(a=0)$ black holes in the large-mass regime [3] (Here $e$ and $m_{e}$ are respectively the electric charge and proper mass of the positron)

$M \gg \frac{e \hbar}{\pi m_{e}^{2}}$,

the emission of charged massive fields is exponentially suppressed as compared to the emission of neutral massless (electromagnetic and gravitational) fields [3,4]. It was therefore argued by Page [3,4] that, by emitting neutral fields which reduce the black-hole mass (without reducing its electric charge), a charged Reissner-Nordström black hole can approach arbitrarily close to the zero-temperature (extremal) limit $T_{\mathrm{BH}} \rightarrow 0$ [It is worth noting that it has been claimed in the physics literature that extremal black holes possess some peculiar physical properties. For instance, it was argued that, despite having non-zero surface areas, the entropy of extremal black holes is zero [5] (see also [6] and references therein)].

The main goal of the present compact paper is to reveal the fact that near-extremal Reissner-Nordström black holes in the regime $T_{\mathrm{BH}} \lesssim \hbar^{2} / G^{2} M^{3}$, if they exist, may violate, through the Hawking emission process, the black-hole condition $Q \leq M$ which is imposed by the Penrose cosmic censorship conjecture [7-9]. This fact, to be proved below, suggests that, in order to guarantee the validity of the fundamental Pen- 
rose cosmic censorship conjecture, the Bekenstein-Hawking temperature of a given mass black hole should be bounded from below by the simple relation $T_{\mathrm{BH}} \gtrsim \hbar^{2} / G^{2} M^{3}$.

\section{The semi-classical Hawking evaporation process of near-extremal Reissner-Nordström black holes}

In the present section we shall analyze the physical and mathematical properties which characterize the Hawking radiation spectra of near-extremal Reissner-Nordström black holes in the regime [The requirement $\Delta \geq 0(M \geq Q)$ follows from the Penrose cosmic censorship conjecture [7-9] as applied to charged Reissner-Nordström black-hole configurations]

$0 \leq \Delta \equiv M-Q \ll M$.

Here $\Delta$ is the excess energy of the charged ReissnerNordström spacetime above the minimal mass (extremal) black-hole configuration with $M=M_{\min }(Q)=Q$. As explicitly shown in [3], the semi-classical decay of nearextremal Reissner-Nordström black holes in the large-mass regime (3) is dominated by the emission of massless neutral photons with unit angular momentum (In particular, it has been shown in $[3,4]$ that, in the large-mass regime (3), the black-hole emission of charged massive fields is exponentially suppressed as compared to the Hawking emission of neutral massless fields. In addition, for the near-extremal black holes (4), the emission of electromagnetic and gravitational field quanta with larger values of the angular momentum parameter $(l>1)$ is suppressed, as compared to the emission of photons with unit angular momentum $(l=1)$, by several factors of the large dimensionless ratio $M / \Delta[3,4])$.

The black-hole radiation power for one bosonic degree of freedom is given by the semi-classical Hawking integral relation [10-13]

$P=\frac{\hbar G}{2 \pi} \sum_{l, m} \int_{0}^{\infty} \frac{\Gamma \omega}{e^{\hbar \omega / T_{\mathrm{BH}}}-1} d \omega$,

where $\{l, m\}$ are the angular (spheroidal and axial) harmonic indices which characterize the emitted field mode, and the frequency-dependent dimensionless parameters $\Gamma=\Gamma_{l m}(\omega)$ are the greybody factors which characterize the linearized interaction (scattering) of the field mode with the curved black-hole spacetime [10-13].

It is interesting to point out that the characteristic thermal factor $\omega /\left(e^{\hbar \omega / T_{\mathrm{BH}}}-1\right)$ that appears in the semi-classical expression (5) for the Hawking radiation power implies that the black-hole-field emission spectra peak at the character- istic dimensionless frequency

$\frac{\hbar \omega^{\text {peak }}}{T_{\mathrm{BH}}}=O(1)$

Since the semi-classical Bekenstein-Hawking temperature (1) of a near-extremal Reissner-Nordström black hole in the regime (4) is characterized by the strong dimensionless inequality (Note that near-extremal Reissner-Nordström black holes are characterized by the strong inequality $\left(r_{+}-\right.$ $\left.r_{-}\right) / r_{+} \ll 1$. This inequality implies the dimensionless relation $G M T_{\mathrm{BH}} / \hbar \ll 1$ [see Eq. (1)] for the near-extremal black holes)

$\frac{G M T_{\mathrm{BH}}}{\hbar} \ll 1$,

one deduces from (6) that, for near-extremal black holes in the regime (7), the characteristic field frequencies which constitute the Hawking black-hole emission spectra are characterized by the strong dimensionless inequality

$M \omega^{\text {peak }} \ll 1$.

Interestingly, and most importantly for our analysis, it has been demonstrated in [3] that, in the low-frequency regime (8) which dominates the emission spectra of the near-extremal black holes, the dimensionless greybody factors $\Gamma_{\operatorname{lm}}(\omega)$ that appear in the integral relation (5) can be expressed in a closed analytic form. In particular, one finds the simple expression [3]

$\Gamma_{1 m}=\frac{1}{9} \epsilon^{8} v^{4}\left(1+v^{2}\right)\left(1+4 v^{2}\right) \cdot[1+O(M \omega)]$

for the characteristic greybody factor of unit $(l=1)$ angular momentum photons [It is worth emphasizing again that, as explicitly shown in [3], these unit angular momentum photons dominate the Hawking radiation spectra of the nearextremal Reissner-Nordström black holes in the large-mass regime (3)], where here we have used the dimensionless physical quantities

$\epsilon \equiv \frac{r_{+}-r_{-}}{r_{+}} \quad$ and $\quad v \equiv \frac{\hbar \omega}{4 \pi G T_{\mathrm{BH}}}$

Substituting Eq. (9) into Eq. (5), and using the relations [see Eqs. (1), (4), and (10)]

$\epsilon=\sqrt{\frac{8 \Delta}{M}} \cdot[1+O(\epsilon)]$ and $\omega=v \sqrt{\frac{8 \Delta}{M^{3}}} \cdot[1+O(\epsilon)]$

one obtains the simple expression [3] 
$P=\frac{\hbar \epsilon^{10}}{3 \pi G M^{2}} \int_{0}^{\infty} \mathcal{F}(v) d v$ with $\mathcal{F}(v) \equiv \frac{4 v^{9}+5 v^{7}+v^{5}}{e^{4 \pi v}-1}$

for the semi-classical Hawking radiation power of the nearextremal Reissner-Nordström black holes.

From Eq. (12) one finds that the function $\mathcal{F}(v)$, which determines the energy distribution of the radiated field modes, has a maximum at

$v=v_{\text {peak }} \simeq 0.511$,

implying that the characteristic photons in the emission spectra of the near-extremal black holes have an energy which is of the order of [see Eqs. (11) and (13)]

$E=\hbar \omega=\hbar G^{-1} v_{\text {peak }} \sqrt{\frac{8 \Delta}{M^{3}}}$.

The Hawking emission of these neutral field modes would reduce the black-hole mass (without reducing its charge) by $\Delta M=-E$. Thus, the mass of the resulting black-hole configuration (after the emission of a characteristic Hawking photon) is given by [see Eqs. (4) and (14)]

$M_{\text {new }}=M-E=Q+\Delta-E$.

Taking cognizance of the Penrose cosmic censorship conjecture [7-9], one immediately realizes that, in order for the Hawking evaporation process to respect the black-hole condition (4), the characteristic energy of the emitted photons must be bounded from above. In particular, using the constraint

$Q \leq M_{\text {new }}$

on the physical parameters which characterize the black-hole configuration after the emission of a characteristic Hawking photon, one finds the lower bound [see Eqs. (14), (15), and (16)]

$\Delta \geq \frac{8\left(\hbar v_{\text {peak }} / G\right)^{2}}{M^{3}}$

on the excess energy of a given mass Reissner-Nordström black hole.

Interestingly, using the relation [see Eqs. (1), (10), and (11)]

$T_{\mathrm{BH}}=\hbar G^{-1} \sqrt{\frac{\Delta}{2 \pi^{2} M^{3}}} \cdot[1+O(\epsilon)]$, one finds from (17) the lower bound

$T_{\mathrm{BH}} \geq \frac{2 v_{\text {peak }} \hbar^{2}}{\pi G^{2} M^{3}}$

on the Bekenstein-Hawking temperature of the near-extremal black holes. Thus, as opposed to the claim made in $[3,4]$, a near-extremal black hole cannot radiate its excess energy all the way down to $T_{\mathrm{BH}} \rightarrow 0$. In particular, our analysis has revealed the intriguing fact that Reissner-Nordström black holes that violate the relation (19) would also violate, through the Hawking evaporation process, the fundamental Penrose cosmic censorship conjecture [7-9].

\section{Summary and dscussion}

In the present compact paper we have raised the following physically interesting question: Can the BekensteinHawking temperature of a given mass black hole be made arbitrarily small? In order to address this question, we have analyzed the physical and mathematical properties of the semi-classical Hawking radiation spectra which characterize near-extremal [see (4)] Reissner-Nordström black holes in the large-mass regime (3).

It has been shown that the black-hole evaporation process may turn a near-extremal black hole with $\Delta<$ $8\left(\hbar \nu_{\text {peak }} / G\right)^{2} / M^{3}$ [or equivalently, with a BekensteinHawking temperature of $T_{\mathrm{BH}}<2 v_{\text {peak }} \hbar^{2} / \pi G^{2} M^{3}$ ] into an horizonless naked singularity with $Q>M$, thus violating the cosmic censorship conjecture [7-9].

Our analysis therefore suggests that one of the following physical scenarios should be valid in a self-consistent quantum theory of gravity:

(1) The Penrose cosmic censorship conjecture can be violated within the framework of a quantum theory of gravity.

(2) The Hawking semi-classical treatment of the black-hole evaporation process breaks down in the regime $T_{\mathrm{BH}} \lesssim$ $\hbar^{2} / G^{2} M^{3}$ of near-extremal black holes in such a way that the emission of charged massive fields (which reduce the black-hole charge and thus increase the black-hole temperature) dominates over the emission of neutral massless fields.

(3) Near-extremal black holes with $\Delta \lesssim \hbar^{2} / G^{2} M^{3}$ do not exist within the framework of a self-consistent quantum theory of gravity. If true, this last scenario implies that the Bekenstein-Hawking temperature of quantized black holes is bounded from below by the simple relation [Note that the dimensionless factor $2 v_{\text {peak }} / \pi$ in Eq. (19) is of order unity. See [14] for an independent physical argument for the existence of a quantum lower bound on the 
Bekenstein-Hawking temperature of black holes]

$$
T_{\mathrm{BH}} \gtrsim \frac{\hbar^{2}}{G^{2} M^{3}}
$$

Acknowledgements This research is supported by the Carmel Science Foundation. I thank Don Page for interesting correspondence. I would also like to thank Yael Oren, Arbel M. Ongo, Ayelet B. Lata, and Alona B. Tea for stimulating discussions.

Open Access This article is distributed under the terms of the Creative Commons Attribution 4.0 International License (http://creativecomm ons.org/licenses/by/4.0/), which permits unrestricted use, distribution, and reproduction in any medium, provided you give appropriate credit to the original author(s) and the source, provide a link to the Creative Commons license, and indicate if changes were made.

Funded by SCOAP ${ }^{3}$.

\section{References}

1. J.D. Bekenstein, Phys. Rev. D 7, 2333 (1973)

2. S.W. Hawking, Commun. Math. Phys. 43, 199 (1975)
3. D. Page, arXiv:hep-th/0012020

4. D. Page, Private communication (2018)

5. S.W. Hawking, G.T. Horowitz, S.F. Ross, Phys. Rev. D 51, 4302 (1995)

6. S. Hod, Phys. Rev. D 61, 084018 (2000). arXiv:gr-qc/0004003

7. S.W. Hawking, R. Penrose, Proc. R. Soc. Lond. A314, 529 (1970)

8. R. Penrose, Riv. Nuovo Cimento I 1, 252 (1969)

9. R. Penrose in General Relativity, an Einstein Centenary Survey, eds. by S.W. Hawking, W. Israel (Cambridge University Press, Cambridge, 1979)

10. D.N. Page, Phys. Rev. D 13, 198 (1976)

11. W.H. Zurek, Phys. Rev. Lett. 49, 1683 (1982)

12. D. Page, Phys. Rev. Lett. 50, 1013 (1983)

13. J.D. Bekenstein, Phys. Rev. Lett. 70, 3680 (1993)

14. S. Hod, Phys. Lett. B 759, 541 (2016). arXiv:1701.00492 